\title{
ILCEA
}

Revue de l'Institut des langues et cultures

d'Europe, Amérique, Afrique, Asie et Australie

$28 \mid 2017$

Passages, ancrage dans la littérature de voyage

\section{Cuba depuis le début de la Période spéciale : diff érents voyageurs pour de multiples impacts}

Cuba since the beginning of the Special period: different travelers for multiple impacts

Janice Argaillot

\section{CpenEdition}

Journals

Édition électronique

URL : http://journals.openedition.org/ilcea/4179

DOI : 10.4000/ilcea.4179

ISSN : 2101-0609

Éditeur

UGA Éditions/Université Grenoble Alpes

Édition imprimée

ISBN : 978-2-84310-374-2

ISSN : 1639-6073

Référence électronique

Janice Argaillot, «Cuba depuis le début de la Période spéciale : différents voyageurs pour de multiples impacts », ILCEA [En ligne], 28 | 2017, mis en ligne le 06 mars 2017, consulté le 30 avril 2019. URL http://journals.openedition.org/ilcea/4179; DOI : 10.4000/ilcea.4179

Ce document a été généré automatiquement le 30 avril 2019

(c) ILCEA 


\section{Cuba depuis le début de la Période spéciale : différents voyageurs pour de multiples impacts}

Cuba since the beginning of the Special period: different travelers for multiple impacts

Janice Argaillot

1 La disparition du camp socialiste a entraîné de nombreux bouleversements pour Cuba, tant sur le plan économique que politique. Le gouvernement de l'île décréta officiellement en 1991 le début de "la Période spéciale en temps de paix », série de mesures destinées à sauver l'économie du pays - et donc à sauvegarder le système révolutionnaire - parmi lesquelles s'imposa très vite l'ouverture au tourisme. En effet, si le tourisme avait auparavant suscité la suspicion des autorités, puisqu'il pouvait supposer l'entrée sur le territoire d'idées contre-révolutionnaires, il devint subitement une solution à la crise, amenant dès la fin des années 1990 plus de bénéfices financiers que des secteurs traditionnels de l'économie cubaine, tels que la production de tabac.

2 Nous nous proposons donc d'analyser la variété des tourismes offerts par Cuba, et leurs différents impacts pour la population depuis le début des années 1990, en nous appuyant notamment - mais pas uniquement - sur l'étude de brochures et guides touristiques, essentiellement francophones. Notre questionnement principal demeurera celui de la rencontre culturelle entre le touriste et la population locale, afin de déterminer si le visiteur perçoit l'île comme un lieu de passage ou au contraire d'ancrage. En effet, l'offre touristique cubaine s'est considérablement étoffée au cours des deux dernières décennies, et nous nous interrogerons tout d'abord sur la façon dont ces différents tourismes ont été introduits dans la société cubaine, mais également sur le voyage réellement proposé au visiteur, et sur la possibilité qui lui est offerte de sortir des sentiers battus lors de son séjour. Dans un deuxième temps, nous nous attacherons aux aspects socio-économiques du tourisme, car si cette activité a supposé un afflux de devises et a effectivement été bénéfique pour l'économie du pays, elle a en parallèle signifié la construction d'une 
double société, ou société à deux vitesses, évidemment peu compatible avec les idéaux égalitaires de la Révolution. Enfin, nous envisagerons l'ouverture au tourisme sous un aspect plus politique, en nous demandant si le touriste constitue une fenêtre ouverte sur le monde, s'il est un représentant d'autres systèmes politiques, ou encore un simple " consommateur ", visiteur de passage avec lequel les Cubains n'ont que peu d'échanges - ce qui interdirait bien entendu tout ancrage culturel.

\section{Un tourisme aux multiples visages}

Pour revenir brièvement sur l'histoire du tourisme à Cuba, nous pouvons dire que l'île fut tout d'abord longtemps considérée comme un lieu de loisirs, et même de tourisme sexuel pour les États-Unis. En effet, après son indépendance vis-à-vis de l'Espagne, Cuba s'est convertie en un centre de plaisirs divers pour les citoyens des États-Unis, état de fait que la Révolution devait venir bouleverser (Michel, 2006: 162). Ainsi, après la victoire des troupes révolutionnaires, le tourisme national fut encouragé et le tourisme international délaissé, ce qui permit à l'île non seulement de conserver ses valeurs et traditions culturelles, mais également de les redécouvrir. En effet, la première décennie de la Révolution cubaine, en ouvrant la voie au tourisme national, a encouragé les Cubains à rencontrer les différentes cultures de l'île, permettant ainsi la consolidation d'un sentiment d'identité nationale ardemment défendu par les autorités, ainsi que la préservation des valeurs morales et « sociales ». Cependant, la crise subie par le Crocodile vert avec la disparition du camp socialiste l'a conduit, dans les années 1990, à s'ouvrir davantage au tourisme international, et à ne plus se cantonner à un tourisme que d'aucuns qualifient de politique.

Précisons que le Bureau national de statistique et information de la République de Cuba ( Oficina Nacional de Estadística e Información, ONEI) diffuse sur son site internet de nombreuses informations concernant l'arrivée de touristes étrangers sur le sol cubain. Ainsi, en janvier 2016, les principaux visiteurs de l'île étaient les Canadiens (au nombre de 173 727), suivis par les Italiens (23 009), les Allemands (21 473) et les Français (19 140)․․ L'Espagne et l'Argentine constituent également deux foyers émetteurs de touristes vers Cuba, bien que leurs difficultés économiques ces dernières années aient entraîné des baisses significatives du nombre de séjours dans l'île, et le nombre d'États-Uniens est quant à lui en constante augmentation (100 000 visiteurs entre janvier et avril 2016). Dès 2013, le seuil des trois millions de visiteurs était franchi, demandant de nouveaux investissements et provoquant toujours plus de changements pour la population.

L'ONEI distingue généralement le «touriste » de l'« excursionniste », visiteurs de passage dont font partie les croisiéristes. Du côté du visiteur, le premier tourisme associé dans l'inconscient collectif à Cuba pourrait être qualifié de tourisme "de sol y playa ». Celui-ci n'induit que peu de relations entre visiteurs et Cubains, et ses conséquences sont avant tout économiques - point sur lequel nous reviendrons plus en avant. La Caraïbe dans sa totalité jouit d'un climat propice au tourisme balnéaire, mais, au sein de l'espace caribéen, Cuba s'est promue et a été promue comme «le » pôle d'attraction bénéficiant d'un ensoleillement majeur, ainsi que d'une sécurité maximale pour le touriste. Le repos et la détente apparaissent bien sûr comme les deux objectifs principaux du séjour, et la découverte culturelle semble donc passer au second plan. D'ailleurs, l'ouverture rapide de l'île au tourisme dans les années 1990 a supposé une construction tout aussi prompte d'infrastructures capables d'accueillir les touristes. Ainsi, à Cuba comme dans d'autres 
pays tropicaux, le tourisme de masse est symbolisé par les « instant resorts ». La rapidité de construction des lieux, reflet de la vitesse avec laquelle le tourisme a envahi l'île, suppose une relation biaisée car uniquement basée sur le commerce, et l'on ne voit pas ici de quelle façon le touriste pourrait dépasser le cliché pour aller vers une véritable rencontre culturelle avec la population locale.

Mais si l'île possède effectivement de majestueux sites balnéaires, il ne s'agit pas là de sa seule ressource. Les sites historiques et architecturaux mis en avant sont principalement ceux de La Havane, et dans une moindre mesure ceux de Santiago de Cuba, même si depuis plusieurs années, le gouvernement cubain cherche à rediriger les flux touristiques, vers Trinidad par exemple. En effet de nos jours, Cuba, tout comme l'ensemble de la Caraïbe, se trouve dans l'obligation de " désengorger » les pôles de tourisme de masse qui s'étaient développés jusqu'alors, afin de tenter de résoudre les problèmes écologiques et énergétiques engendrés par ce même tourisme (consommation en eau et électricité, pollution...).

7 Les tourismes alternatifs alors proposés, écotourisme, tourisme de santé (qui stimule la recherche et le développement de médicaments et thérapies) et thermalisme, tourisme historique (lié en bonne partie au « legs » de la période esclavagiste), proposent une autre vision de Cuba. Et il faut effectivement «souligner l'expérience cubaine en matière de tourisme de santé [...]» (Raboteur, 2009a: 86), ou les nombreuses activités promues depuis quelques années à Cuba : pêche, chasse, équitation, spéléologie, tourisme " vert " ou « écologique », par le biais des organismes Cubamar et Gaviota (Michel, 1998 : 277). Ces activités sont couplées à une offre portant sur le tourisme linguistique et culturel, les touristes pouvant opter pour des cours de langue ou des circuits retraçant l'histoire de lîle.

8 En conséquence, le tourisme a été le moteur d'une réorientation de la formation professionnelle et universitaire à Cuba, l'île ne pouvant se permettre l'amateurisme en matière de tourisme culturel. Le système éducatif met donc l'accent, dans le domaine du tourisme, sur la formation de personnel qualifié (études en management, économie, langues...). Cela étant, on peut se demander si les circuits historiques et culturels promus ne cherchent pas à satisfaire encore et toujours les attentes du touriste. En effet, la construction d'établissements de restauration au beau milieu d'anciens baraquements d'esclaves dans le sud-est de l'île fait craindre à de nombreux experts des dommages irréversibles pour les bâtiments historiques, qui deviennent finalement la scène d'une pièce de théâtre dans laquelle les Cubains jouent des rôles, devant un public constitué de visiteurs.

Dans la même veine, il faut souligner que les conséquences sur la faune et la flore de la construction massive d'hôtels de luxe sur les plages de certaines villes (comme à Varadero par exemple) sont absolument désastreuses. Malgré tout, le gouvernement de l'île a contribué, peut-être sans le vouloir, à sauvegarder la biodiversité, en orientant les flux touristiques vers des points précis, et en évitant donc toute dégradation de nombreux sites et paysages ; ceci est tout particulièrement vrai pour la barrière de corail, au large de l'île de la Jeunesse, encore appelée île des Pins (Lamic, 2008 : 105).

On voit ici à quel point le tourisme induit des situations paradoxales dans l'île, et l'observateur peut rapidement penser que les multiples facettes du tourisme à Cuba constituent une chance. Mais la participation du peuple cubain au développement de tourismes alternatifs, desquels naissent de nouvelles opportunités économiques, suppose que les bénéfices engendrés soient répartis, réinvestis, et redistribués aux acteurs de ces 
nouveaux tourismes, et en premier lieu aux populations locales. En plus de maintenir les communautés dans leurs lieux de vie et environnements originels, les tourismes alternatifs permettent alors d'exploiter les ressources, de mettre en valeur le patrimoine, mais également de le sauvegarder, l'argent dépensé par le touriste dans le pays visité permettant la restauration d'édifices et l'entretien de certains lieux - tel est par exemple le pari du «Plan maestro de rehabilitación integral de la Habana Vieja » dirigé par le Bureau de l'Historien de la Ville.

11 En outre, le tourisme culturel soulève en lui-même un certain nombre de questions. On a pu souligner le dynamisme insufflé à l'artisanat par la mise en valeur du tourisme culturel, et en effet, l'arrivée de touristes désireux de rentrer chez eux les bras chargés de souvenirs a entraîné une réactivation - sans doute pas un renouveau - dans ce domaine. On a ainsi vu se multiplier les échoppes d'artisans souhaitant profiter de la manne financière sous-tendue par le tourisme : «Il est incontestable d'affirmer que concernant l'artisanat, l'influence du tourisme a été bénéfique. Il est évident de dire que cette soif de voir, d'entendre, d'écouter et d'acheter du touriste a stimulé la demande dans les domaines de l'art visuel, de l'art folklorique et de la conservation du patrimoine » (Raboteur, 2009b : 67-68). Ainsi, le tourisme culturel permet-il la survivance de pratiques et traditions, autrement dit du lien identitaire qui unit les communautés, dès lors que le visiteur montre un intérêt pour le patrimoine du pays visité. Encore faut-il que l'artisanat ne concentre pas son offre sur la production de souvenirs uniquement «typiques » pour l'imaginaire européen ou occidental, à travers des objets « attendus » (cendriers à l'effigie du Che, objets décoratifs aux couleurs d'une célèbre marque de rhum...).

Car le souvenir ramené d'un séjour n'est bien souvent « un petit bout de la culture de l'autre » que dans l'esprit du voyageur. Cuba est à ce sujet le parangon du pays stéréotypé à travers les souvenirs qui y sont vendus: "Que rapporter de Cuba? Naturellement, du rhum et des cigares " (Leroux Monet, 2008: 32) nous dit-on dans bon nombre de guides touristiques. Ainsi, dans son édition de 2010 sur Cuba, «Le Petit Futé » donne dès les premières pages comme mots-clés concernant l'île des vocables comme "cigares » ou « cocktails » (Auzias, Labourdette, 2010:21), et ouvre sa section « Arts et culture » par un article long de trois pages sur les cigares. Bien que l'on précise à la suite que l'île offre de nombreux souvenirs «culturels» (livres et disques notamment), l'adverbe «naturellement » couplé à la position première du rhum et des cigares sur la liste des souvenirs "à rapporter » offre matière à analyse. L'image de fête tropicale permanente associée à l'île apparaît ici, et ne pas rapporter les deux produits phares précités apparait presque comme une faute de goût. Clairement, on insinue dans l'esprit du touriste l'idée qu'il ne sera pas perçu comme un "véritable » voyageur s'il ne ramène pas ce qui est présenté comme «typiquement cubain ». Parmi les guides touristiques français consultés par nos soins, seuls les volumes publiés chez «Lonely planet " présentent de manière poussée le patrimoine cubain (depuis l'architecture jusqu'à la faune et la flore), emmenant le visiteur «au-delà des plages », évoquant par le terme "folklore » les traditions culturelles héritées des esclaves, et indiquant dans une section intitulée «Cuba, la vraie» des lieux de visite culturels - une section «farniente total» offrant un cloisonnement clair de l'offre touristique.

Il convient encore de souligner que les «manifestations spirituelles sont particulièrement pratiquées et suivies » dans la Caraïbe, à l'instar du vaudou en Haïti ou de la santería à Cuba (Raboteur, 2009b : 69). Cuba offre effectivement un tourisme "religieux ", qui peut devenir dangereux dès lors qu'il tend à satisfaire une demande plutôt qu'à transmettre 
des valeurs culturelles. En effet, cette sorte de tourisme permet sans aucun doute la protection d'une partie du patrimoine culturel cubain, car des pratiques religieuses délaissées, voire méprisées, retrouvent grâce à l'intérêt des voyageurs un statut «honorable». Mais cela peut conduire à effacer une partie de l'identité culturelle intrinsèque à ces pratiques : « En 1993, l'État a ouvert plusieurs sites touristiques offrant objets et spectacles inspirés du corpus afro-cubain comme le Bazar des Orishas à Guanabacoa. Des santeros et de babalaos auraient même été contactés par les autorités pour y pratiquer des consultations en dollars pour les étrangers » (Bastian, 2007 : 165). Ainsi, si les pratiques religieuses cubaines, en tant qu'éléments culturels, peuvent attirer les touristes, elles peuvent également devenir monnayables, et n'être plus alors qu'un élément commercial. Dans le cas de Cuba, on peut en arriver à des phénomènes presque antithétiques, puisque l'on n'hésite pas à allier pratique ancestrale et technologie moderne dans le but de satisfaire les touristes :

À La Havane, l'Association culturelle yoruba de Cuba, reconnue par l'état en décembre 1991, occupe un immeuble colonial désaffecté rénové grâce à un prêt public. Depuis 1995, il sert de "musée-temple interactif» des Orishas, avec boutique, restaurant, galerie d'art, salle de conférence et bibliothèque qui sont inclus dans les circuits touristiques. Le cas cubain est exemplaire de la manière dont l'état peut instrumentaliser la religion à des fins touristiques dans un contexte où l'industrie touristique se trouve sous sa tutelle. (Bastian, 2007 : 165)

14 L'instrumentalisation des pratiques culturelles et la diffusion de stéréotypes concernant la culture locale ne permet donc pas au voyageur de s'immerger réellement dans le quotidien des Cubains. Il s'agit d'un réel danger pour la construction et la transmission de l'image de l'île, car nombre de brochures touristiques laissent entendre que le peuple cubain n'a d'autre préoccupation ou de besoin que de faire la fête - il en paraitrait presque oisif - et l'on note même que ses manifestations culturelles sont converties en futilités :

[...] un magazine intitulé Voyages et exotisme présente un numéro sur « Cuba, la perle des Antilles ». Lisons quelques lignes de l'éditorial: Le peuple, amène et langoureux, vit de sourires, de danses et de musique. Sa joie lascive et langoureuse n'occulte pas, malgré sa détermination et sa passion de vivre, une grande valeur humaine, une haute valeur morale. L'accueil est généreux, direct et franc, et le sentiment de sécurité total. [...] C'est un voyage de l'âme aux rives très sûres de la splendeur et du rêve ». Va pour la sécurité pour le touriste [...] mais existe-t-il seulement un peuple sur terre capable de vivre "de sourires, de danses et de musique?» (Michel, 1998 : 276-277)

15 L'interculturalité prend donc un sens particulier lorsqu'elle devient un argument de vente, et lorsque des expressions simplistes, présentées comme des vérités que personne n'oserait nier, se convertissent en explications. En d'autres termes, nous sommes en présence d'un cercle vicieux, au sein duquel les préjugés et la méconnaissance débouchent sur un tourisme qui n'est pas un tourisme de la découverte ou de la rencontre culturelle. D'ailleurs, dans de très nombreux guides francophones, la Caraïbe dans sa totalité semble se résumer à l'architecture coloniale havanaise et à la plage de Varadero. Pourrait-on parler de paresse intellectuelle pour expliquer ce manque d'envie de sortir des sentiers battus et d'aller vers « l'Autre»? 


\section{Conséquences socio-économiques}

16 Le tourisme a incontestablement été synonyme d'entrée de devises sur le territoire cubain, et a en partie permis de faire face à la crise totale consécutive à l'effondrement du bloc socialiste. Mais il faut ajouter qu'il a également contribué à la création d'une société à deux vitesses au sein de l'île de la Révolution, et a instillé une certaine jalousie entre ses habitants, puisque les retombées économiques du tourisme n'ont pas nécessairement touché de la même manière tous les Cubains. Le tourisme a donc favorisé une division, voire une fracture au sein de la population cubaine : «À Cuba, on appelle ce phénomène la "pyramide inversée". Une large part de la population la moins éduquée gagne plus d'argent que les traditionnelles élites. [...] Ce phénomène a lieu dès lors qu'il existe une disparité trop importante entre les salaires des personnes travaillant dans le secteur du tourisme et le reste de la population locale» (Lamic, 2008: 74). Ainsi, on note les modifications profondes dans l'inconscient collectif cubain, qui font que les «élites traditionnelles » ne le sont désormais plus. On observe dans le même temps les changements sociétaux, dès lors que de nombreux Cubains abandonnent leur emploi de professeur ou médecin, pour devenir chauffeurs de taxi ou serveurs afin de profiter de la manne financière que suppose le tourisme.

L'économie de l'île basée sur le dollar puis la création du CUC ont en effet conduit les Cubains à s'impliquer très directement dans le tourisme. Aujourd'hui, les Cubains travaillant au contact des touristes sont effectivement payés en CUC, devise convertible indexée sur le dollar états-unien, que le gouvernement de Raúl Castro cherche à éliminer depuis plusieurs mois, au profit d'une monnaie unique. Une fois le carnet de rationnement (libreta) épuisé, la seule façon de se procurer certaines denrées alimentaires et des produits d'hygiène se trouve dans les magasins qui vendent en CUC, et non en monnaie nationale. En conséquence, les différences de salaire (pourboires compris) et de conditions de vie entre les Cubains ayant une activité professionnelle liée au tourisme et les autres sont énormes. On voit d'ailleurs s'ouvrir de nombreux paladares, sorte de petites auberges tenues par des particuliers au sein même de leur maison, ne pouvant accueillir plus de vingt convives, que fréquentent les touristes mais également depuis peu quelques Cubains. Ainsi, tout est mis en place pour que les Cubains fréquentant les touristes se convertissent en une classe "à part ", en une petite bourgeoisie au sein de la société révolutionnaire - antithèse s'il en est - provoquant l'ire des Cubains qui restent en marge (Michel, 1998 : 286-287).

Le tourisme a en outre conduit à la prolifération d'un phénomène appelé «jineterismo » (" jinetera " pouvant être traduit en français par «cavalière»). Depuis le début des années 1990, la prostitution (qui avait presque totalement disparu), ressurgit «dans certaines grandes villes portuaires, à La Havane en particulier. Il ne s'agit plus d'un phénomène social, tel qu'il existait encore dans les premières années de la Révolution. Il résulte plutôt de la politique marquée de Cuba en faveur du tourisme international» (Gay-Sylvestre \& Estrade, 2006 : 191). On peut donc voir aujourd'hui des jeunes hommes ou de jeunes femmes « escorter » des touristes durant leur voyage, ce qui n'est pas sans risque pour eux, puisque les autorités font officiellement preuve d'une grande sévérité avec les Cubains reconnus coupables de prostitution. Quoi qu'il en soit, le tourisme et ses corollaires peuvent entraîner la suspicion et la jalousie, et «même la séduction se fait troublante et conspiratrice, comme l'indique [...] cet extrait de la romancière cubaine en 
exil Zoé Valdés : "Ceux qui ont des dollars, ce sont les prostituées, les délinquants, et aussi les rares employés, mal payés, des entreprises mixtes. Les riches, ce sont les étrangers." " (Michel, 1998 : 272). Il faut tout de même dire que certains guides mettent en avant le tourisme sexuel non comme une caractéristique intrinsèque de la culture cubaine, mais plutôt comme une "dérive " (Simoni, 2011: 207) due à la crise économique. D'autres appellent le visiteur à se méfier des jineteros, ou à voir plus loin que l'image de détresse qu'ils renvoient :

Même si les choses vont rarement plus loin (peu de vols purs et simples ou d'agressions), vous trouverez vite désagréables ces sollicitations parfois incessantes, qui ne débouchent que très rarement sur de vraies rencontres [...]. Sachez être ferme, [...] d'autant que vous aurez bien d'autres occasions de lier de vraies amitiés, en faisant vous-même le premier pas. (Géoguide, 2014 : s. p.)

Comme toujours, Cuba semble diffuser l'image d'une île particulière. En effet, il convient de noter l'aspect "exubérant» qui caractérise l'île dans l'imaginaire collectif et la transforme en un espace festif pour le touriste. Il faut reconnaître que l'exubérance est une constante dans les brochures touristiques évoquant Cuba, tout comme la bonhommie et le caractère sympathique des Cubains :

Dans son analyse de discours de documents et de publicités touristiques sur Cuba, l'anthropologue Michel répertorie [...] les fréquents éloges de l'hospitalité, de la gentillesse et de la joie de vivre des Cubains. Cet auteur relève également les références récurrentes au caractère " amène et chaleureux » du peuple cubain [...]. Le registre sémantique de ces exemples met en avant la nature exubérante, passionnelle et sensuelle des Cubains, et montre plus directement encore l'importance de l'amour à Cuba. De telles images [...] persistent aujourd'hui, et on les retrouve dans plusieurs guides de voyage destinés au grand public (Simoni, 2011 : 202-203).

Nous insisterons ici sur un point: la "passion » des Cubains n'est semble-t-il que le pendant de leur "sensualité ", et est surtout à relier à la sexualité. Le Cubain ne saurait donc être passionné par autre chose que l'amour physique, et « le célèbre Guide du routard [dans ses éditions en italien de 2005 et en français de 2007] consacre par exemple une section spéciale à l'Amor à Cuba. L'amour y est qualifié de sport national, au bon sens du terme, une activité quasi vitale pour les Cubains, un loisir conjugué sous toutes ses formes. Plus encore, Cuba y est considéré comme probablement le pays le plus sensuel de la terre, pour ne pas dire sexuel » (Simoni, 2011 : 203). Le touriste ne peut avoir un regard objectif, il est au contraire influencé par cette omniprésence du sexe dans la presse touristique portant sur Cuba, qui affirme d'ailleurs parfois que « la prostitution est chose courante en ville ou dans les stations balnéaires touristiques, d'autant plus qu'elle n'offense personne" (Auzias \& Labourdette, 2016: s. p.). Cette vision dramatique qui réduit le Cubain à l'état animal, le convertit en une bête non plus passionnée mais obsédée, fait craindre que la population cubaine ne soit perçue par le visiteur que comme une prestation incluse dans son forfait. L'image diffusée du Crocodile vert n'est pas celle d'un territoire de culture, de l'ajiaco, c'est-à-dire du foisonnement et du bouillonnement culturel décrit par l'ethnologue cubain Fernando Ortiz; tout sur l'île parait sexué et sexuel, et le corps humain se convertit en appât à touristes :

On note, en parcourant les publicités et autres brochures, une véritable entreprise de mise en tourisme du corps humain, qu'il soit féminin ou masculin. Entre autres exemples, citons Cuba magazine, brochure de l'agence Cubanacan (une des grandes organisations de tourisme et de commerce de Cuba, implantée dans neuf pays) : la couverture montre une jeune fille blonde (apparemment une touriste), dansant avec un bonheur évident, dans les bras d'un grand Noir (apparemment un Cubain). 
[...] Pour attirer les touristes mâles, les brochures peuvent, semble-t-il, faire l'économie du texte, les très nombreuses illustrations et photographies de femmes cubaines se déhanchant en petite tenue suffisent amplement... (Michel, 1998 : 276) cubaines ont parfois mis en avant la présence de la population noire dans l'île pour promouvoir le tourisme, expliquant que le métissage culturel et la Révolution avaient combattu la discrimination raciale, assurant ainsi une certaine tranquillité aux visiteurs Noirs (Goldstone et al., 2003 : 168). Néanmoins, la présence de la population Noire dans l'île n'est jamais expliquée, et le Noir est confiné au rôle de «danseur» ou de vendeur ambulant, relayant ainsi des clichés raciaux et racistes. On note également "l'image persistante de la "femme mulâtre" (mulata) en tant que "fille de l'amour" (Simoni, 2011 : 203) qui est diffusée, en contrepoids de l'image de l'homme « viril », pour qui le machisme serait presque une qualité - et il n'est que de consulter l'illustration de couverture de l'édition 2017 du Petit Futé concernant Cuba pour s'en convaincre: une jeune femme métisse et vêtue "à la créole ", expirant lascivement la fumée d'un cigare, les yeux perdus dans le vide. Ces stéréotypes raciaux et de genre contribuent « à expliquer l'attrait des Caraibes comme destination touristique où le sexe serait facile et même un ingrédient naturel de l'expérience vacancière " (Simoni, 2011: 203). Se rendre à Cuba, « où la sexualité n'est pas un tabou » (Auzias \& Labourdette, 2016 : s. p.), dans le but d'avoir des relations sexuelles avec un autochtone serait donc aussi naturel que d'effectuer un voyage à New York pour voir la Statue de la Liberté. Dans ces conditions, le visiteur n'est qu'un être de passage, et aucun ancrage culturel n'est possible.

uoi qu'il en soit, le tourisme a induit une augmentation des activités commerciales illégales, et en premier lieu du marché noir, que la Période spéciale a réactivé - même si la légalisation récente du cuentapropismo a dans une certaine mesure induit une légalisation de l'économie parallèle. On a en tous les cas constaté un changement de mentalité, et une envie de plus en plus forte chez les Cubains de tirer parti de leurs contacts avec le visiteur. En effet, l'arrivée de touristes venus de pays dits développés n'est pas sans conséquence sur l'attitude des jeunes générations, qui n'ont connu ni le mouvement de guérilla révolutionnaire (1956-1959), ni la période précédente durant laquelle l'̂le était sous le joug du dictateur Fulgencio Batista : « Le tourisme étranger, un mal nécessaire aux dires des dirigeants, est un élément dont l'incidence négative est la plus visible sur les jeunes. Le touriste qui arrive à Cuba, issu en majorité des secteurs moyens des sociétés capitalistes, agit à Cuba en représentant direct de la société de consommation » (Gay-Sylvestre \& Estrade, 2006 : 234-235). Le premier impact du tourisme sur ces jeunes est donc l'envie que fait naître le fait de côtoyer des Européens ou ÉtatsUniens dotés d'un matériel technologique dernier cri ou de vêtements de luxe. Ceci est à tel point vrai qu'au sein du système de débrouille instauré par les Cubains pour résoudre ( resolver ») le quotidien, ceux qui arrivent à voyager, et en premier lieu les personnels navigants de bord des compagnies aériennes, sont devenus un rouage essentiel du contact avec «l'extérieur » :

[...] même si les compagnies aériennes limitent le poids des bagages en classe économique à $20 \mathrm{ou} 23 \mathrm{~kg}$, plusieurs passagers choisissent de payer pour les kilogrammes supplémentaires ou ils prennent la classe « Tropical » sur la Cubana de Aviación, qui leur permet d'emporter $46 \mathrm{~kg}$ de bagages, moyennant quelques 200 dollars par trajet. C'est sans oublier les bagages en cabine. Les équipages de la Cubana de Aviación profitent, eux, de leur bref passage dans un aéroport étranger pour acheter des dizaines et des dizaines de produits. Non sans ironie. Lors de l'hiver 2012, le départ d'un vol de la Cubana de Montréal a ainsi été repoussé de 
plusieurs heures. L'équipage était arrivé en retard après être parti faire des courses. Les mules sont un phénomène très important dans une société cubaine qui manque de tout. À Cuba, l'Occident arrive dans les valises. (Lemieux, 2013 : s. p.) autres appareils numériques ne peut qu'attiser la convoitise des jeunes Cubains, qui se mettent alors à douter des vertus du système politique révolutionnaire, basé certes sur l'égalité, mais surtout sur l'équité dans ce qui leur apparaît comme une certaine pauvreté. En ce sens, le tourisme a provoqué une remise en cause des principes et idéaux révolutionnaires, car si la crise consécutive à l'effondrement du camp socialiste avait provoqué la réapparition de la pauvreté, et avait donc dévoilé une faille dans le système révolutionnaire, le tourisme a recréé des «classes». Effectivement, l'ensemble de la population cubaine ressent nettement la fracture entre son quotidien, et les prestations offertes aux touristes. De la sorte, il apparaît clairement que le tourisme induit non seulement une division sociale, mais également un questionnement pour les Cubains, qui s'interrogent sur leur système politique, et en arrivent à se demander si les acquis de la Révolution en matière de santé ou d'éducation valent les sacrifices consentis. Et il faut dire que les investissements du gouvernement cubain dans le secteur touristique furent plus que conséquents, puisqu'entre 1990 et 1994, 500 millions de dollars furent injectés dans les infrastructures liées au tourisme (Goldstone et al., 2003 : 154).

Dès lors, on comprend que le tourisme soit aussi dangereux que nécessaire pour le gouvernement cubain, et que le dialogue entre les personnes vivant sur l'île et les étrangers ne soit pas spécifiquement promu. Assurément, l'ouverture au tourisme, synonyme d'entrée de devises sur le territoire, peut activer la «dissidence» de la population, confrontée de façon très concrète à des différences de niveaux de vie qu'elle n'est plus encline à accepter. Le contact entre visiteurs et visités paraît donc plus conflictuel que culturel; il semble que la comparaison et l'opposition de systèmes de valeur priment sur le reste. Et c'est bien, encore et toujours, la mise à disposition de l'île pour ses visiteurs qui constitue le point d'achoppement de la relation entre ces derniers et les Cubains :

Un des principaux objectifs que devraient à notre sens prendre en compte les « développeurs » cubains consiste à faire bénéficier davantage la population locale des fruits du tourisme: cela améliorerait d'un côté le pouvoir d'achat des autochtones et de l'autre les relations entre les visiteurs et leurs hôtes. C'est dans cet esprit qu'il conviendrait par exemple de ralentir fortement la consommation de produits importés - le gouvernement mais aussi le peuple cubain n'en tire aucun avantage financier - et de miser autant que possible sur la production locale: concrètement, cela peut se traduire, par exemple, dans les restaurants, par des desserts au menu composés de bananes ou de noix de coco plutôt que de fraises ou de chocolat... (Michel, 1998 : 286)

Le fait de se plier aux exigences et attentes des touristes aggrave la crise morale, et n'améliore pas la condition économique des Cubains, dès lors que le tourisme signifie une augmentation des importations au détriment de la mise en valeur des produits locaux. On en viendrait à croire que, pour préserver une identité cubaine avant tout axée sur la Révolution, les autorités préfèrent faire voyager le visiteur dans un nouvel espace certes, mais empli d'éléments de sa culture d'origine, ou en d'autres termes dans un espace européen ou occidental délocalisé sous les tropiques.

Outre la prostitution, les indicateurs montrent que la criminalité a augmenté à Cuba depuis l'ouverture au tourisme (Colantonio \& Potter, 2006 : 161). Les taux de criminalité 
varient cependant selon les types de tourisme promus par les villes et les quartiers, et si la petite délinquance et les vols à l'arraché ont augmenté dans le quartier du Malecón à La Havane, d'autres quartiers tels que Miramar et son complexe hôtelier Monte Barreto, destiné au tourisme d'affaire, semblent relativement épargnés (Colantonio \& Potter, 2006 : 167). Les Cubains ont également vu apparaître le trafic de drogue, qui pendant des années était resté une activité marginale. Dans le même temps, la mendicité s'est aussi répandue, et l'augmentation de l'insécurité a conduit au renforcement de la présence policière, ce qui ne permet pas précisément une plus grande aisance du contact entre touristes et habitants de l'île.

D'aucuns soulignent en sus que les jeux d'argent ont pris au fil du temps beaucoup d'importance pour certains Cubains, et ont également mis en avant une modification des schémas sociaux et une transformation des "valeurs traditionnelles familiales" (Colantonio \& Potter, 2006 : 166). Ainsi, c'est une modification des codes de la société et de la manière de vivre ensemble que provoque le tourisme. Pour le dire autrement, le voyageur amène avec lui un peu de sa culture, mais également de son mode de vie, et les Cubains s'en imprègnent parfois à tel point qu'ils en oublient leurs propres repères identitaires. À ce sujet, la «modernisation de la position de la femme dans la société » (Colantonio \& Potter, 2006 : 169) induite par le tourisme le convertit en élément positif, et il faut alors se demander si le tourisme n'est, malgré tous ses aspects négatifs, pas un pont entre l'île et le monde.

\section{Touristes et cubains, touristes vs cubains ?}

Nous l'avons dit, les touristes bénéficient dans l'île de conditions que ne connaît pas la population, et il semble au premier abord que de véritables ghettos touristiques aient été créés. Pour le dire autrement, on a dressé des barrières invisibles entre habitants de lî̂le et visiteurs en faisant du tourisme une source de revenus et non une ouverture sur le monde, enfermant chacun dans un rôle prédéfini. D'une certaine façon, les autorités officielles ont tenté de faire se côtoyer deux mondes, en prenant soin d'éviter sinon le contact, à tout le moins l'échange. Et il faut bien dire que le tourisme politique (voyage organisé par des partis politiques ou organisations syndicales dans le but de consolider les liens avec les organisations du pays d'accueil) n'est plus celui qui prédomine, contrairement à ce que l'on pouvait constater jusque dans le milieu des années 1980. En effet, si tout est lié à la politique, en particulier à Cuba, et si les messages à la gloire de la Révolution et de ses grandes figures placardés par le gouvernement dans tous les espaces de la vie publique font qu'aller à Cuba signifie nécessairement se confronter à un système politique spécifique, les touristes internationaux sont désormais peu nombreux à faire le déplacement afin d'aider à la zafra (récolte de la canne à sucre) et soutenir ainsi le « régime ». Des organismes proposent toujours des «voyages solidaires" permettant d'assister aux cérémonies du 1er mai dans l'île, mais il ne s'agit pas de l'offre attirant le plus de visiteurs.

Cela étant, le touriste qui arrive aujourd'hui à Cuba est porteur d'idées et d'idéaux, certains diront d'idéologies, que le gouvernement cubain ne souhaite pas voir se propager dans l'île. Cette tendance ne pourra que s'accentuer à l'avenir, si le dégel des relations avec les États-Unis débouche sur une réelle possibilité pour les citoyens états-uniens de se rendre librement dans l'île. 
30 Mais il faut encore faire une distinction entre touristes séjournant à l'hôtel, et touristes optant pour un séjour «chez l'habitant». En 2011, 16392150 nuitées de touristes étrangers dans les hôtels ont été enregistrées à Cuba (et 3075062 de nuitées pour les touristes nationaux), pour 24571 nuitées chez l'habitant (1 254386 pour les touristes nationaux $)^{2}$. Ces statistiques montrent clairement que le logement en "casas particulares " n'est pas le premier choix des touristes internationaux, ce qui interroge quant à leur volonté de découvrir une Cuba " authentique ».

31 Si prendre un logement chez l'habitant n'est, du point de vue des formalités, pas très compliqué pour le visiteur (et est a fortiori économique, puisqu'il faut compter en moyenne 30 CUC par chambre et par nuit), il implique pour les Cubains qui obtiennent la licence d'«arrendadores» (personnes habilitées à recevoir des touristes en leurs demeures) une surveillance étroite de la part des autorités. L'achat de la licence représente en soi un investissement conséquent, et signifie que son possesseur devra verser chaque mois à l'État un impôt, qu'il ait trouvé un locataire ou non. Pour chaque touriste hébergé, une déclaration doit être remplie, qui donne lieu au passage de policiers le lendemain de l'arrivée des visiteurs, visiteurs qui ne doivent théoriquement jamais être laissés seuls dans la maison ou l'appartement qui les accueille. S'impose alors un « système $\mathrm{D}$ », certains visiteurs n'étant malgré tout pas déclarés - et il faut préciser que le risque d'être dénoncé par un voisin expose le Cubain qui loue sa maison ou son appartement hors de la réglementation officielle à de sévères sanctions. Pour beaucoup, les éventuels bénéfices à louer une pièce ou deux de leur maison ne valent pas la peine de prendre autant de risques et d'affronter une bureaucratie bien souvent sourde à toute négociation.

32 Cependant, les liens qui se créent entre visiteur et hôte, au-delà de l'omniprésence de l'État, induisent une question : le touriste est-il fenêtre ouverte sur le monde, un visiteur de passage, un consommateur? Dans le cas d'un séjour chez l'habitant, il faut rappeler que les CDR (Comités de Défense de la Révolution), que l'on pourrait décrire comme des associations de résidents présentes dans chaque pâté de maison, en charge notamment de surveiller les activités du quartier et les éventuels agissements subversifs des citoyens cubains, n'aident pas ces derniers à se livrer en toute confiance aux visiteurs.

Cela étant, Cuba ne peut qu'être attentive à la qualité de ses prestations touristiques, qui constitueront un point d'appui au "bouche à oreille » la concernant. Ainsi, les autorités cubaines prêtent-elles une attention particulière à la satisfaction du touriste, car c'est bien du ressenti de ce dernier que naîtra le souvenir du voyage effectué - souvenir qu'il transmettra à ses proches, hypothétiques futurs visiteurs. En somme, comme pour tous les autres pays, le touriste qui se rend à Cuba vit une expérience particulière $-a$ fortiori s'il n'est pas logé chez l'habitant -, puisqu'il bénéficie d'attentions qui tendent à faire de son séjour une parenthèse "enchantée ", ce qui ne lui permet pas toujours de dépasser les stéréotypes. D'ailleurs, le boom touristique que connaît actuellement le Crocodile vert laisse à penser que le tourisme chez l'habitant peut lui aussi exploser (les hôtels sont à certaines époques de l'année saturés), induisant encore et toujours la question de la création de liens spontanés et dépourvus d'arrière-pensées entre visiteurs et "visités ". La visite du touriste ne doit pas être perçue comme une intrusion dans la vie des Cubains, ce qui peut être difficile lorsque les deux parties partagent le même logement. En outre, il ne faut pas oublier qu'il s'agit d'une relation commerciale, fondée sur un service en échange d'argent. Cette relation monétaire peut être un poison pour le lien culturel, puisqu'elle induit une relation de domination. On sent le fil ténu entre marchandisation 
de la rencontre et relation authentique permettant au visiteur de laisser une trace (positive ou non) dans l'île et d'en ramener une parcelle dans son pays d'origine. Havanatour, société spécialisée dans le voyage à Cuba dont la maison-mère est située à La Havane, promet ainsi dès la première page de sa brochure «hiver 2016/2017 » à destination des touristes francophones :

Havanatour vous propose de goûter à l'hospitalité légendaire des Cubains. Loger chez l'habitant est une expérience unique qui vous permettra des échanges inoubliables avec vos hôtes et de découvrir Cuba sous un autre angle. Vous pourrez également y déguster une cuisine typique et savoureuse. Pour vous permettre de revenir avec des souvenirs uniques, nous reconduisons le circuit Cubania, alliant nuitées à l'hôtel et chez l'habitant. [...] le repas est un moment propice et particulier pour partager un bout de conversation avec votre hôte.

Ainsi, sauf à être hébergé par un Cubain, le voyageur ne « découvre » pas réellement l'île, dans la mesure où il se transporte à Cuba, mais amène également son mode de vie. Dès lors que le touriste ne sort pas ou très peu de son hôtel, il n'effectue plus un voyage, mais un simple séjour. Il n'y a pas de rencontre culturelle, et le souvenir qu'il ramène avec lui du pays visité n'est qu'une pierre de plus à l'édification de l'image " clichée » de Cuba. On s'aperçoit ainsi que le touriste n'accepte pas toujours de changer le regard qu'il porte sur le pays visité, et que le déplacement n'est pas dans tous les cas un moyen de confronter un savoir théorique apporté par les guides avec sa propre réalité, avec l'expérience concrète et personnelle du terrain.

À ce sujet, un autre point marquant lorsque l'on évoque Cuba en tant que destination touristique est l'image diffusée de la Révolution. Cuba est le plus généralement présentée comme un paradis pour les touristes, mais aussi et surtout comme un enfer pour les Cubains :

Dans une revue qui se propose de "voyager le monde autrement », la «Perle des Caraïbes » est décrite davantage comme un enfer quotidien qu'un paradis oublié, l'accent étant mis sur le castrisme intraitable et l'ampleur de la crise économique. Le tourisme est perçu comme étant plus qu'une bouée de sauvetage: "[les] étrangers sont les bienvenus: ils représentent le dernier salut de la république socialiste ». Voilà qui résume parfaitement une pensée aussi dominante que rassurante en vogue en occident. (Michel, $1998: 275$ )

Le terme "castrisme " est primordial, puisqu'il porte en germe une opposition à un système que beaucoup d'Occidentaux ont du mal à se représenter. Système critiquable, comme le sont tous les autres, la Révolution cubaine attire ou repousse, et devient souvent le moteur du voyage chez le touriste désireux d'en apprendre plus. Quant au tourisme présenté comme "une bouée de sauvetage", il projette l'image d'un pays pauvre et d'une population aux aguets, à l'affût du moindre bénéfice. On se demande alors comment le pays pourrait par la suite être présenté comme «sûr » pour le visiteur, et comment ce dernier pourrait se rendre à Cuba en confiance. D'une certaine façon, on oppose ici les touristes aux Cubains, touristes qui semblent se rendre dans l'île comme pour participer à un "safari de la misère ", et repartir convaincu que son mode de vie habituel comporte de nombreux avantages au regard du système cubain. On oublie alors les acquis de la Révolution cubaine, mondialement reconnus, principalement dans les domaines de l'éducation et de la santé.

Ainsi, le visiteur doit accepter de se départir de sa vision occidento-centrée en arrivant sur l'île. Malgré tout, l'étranger qui visite Cuba et apporte ses valeurs sur l'île ne contribue pas nécessairement à en uniformiser la culture ou à la transformer en parc 
d'attractions. Effectivement, lorsqu'on les interroge, les habitants de l'île déclarent avoir envie de travailler dans le secteur touristique non seulement pour les conditions de travail et les salaires, mais également pour les échanges culturels que les emplois dans ce domaine supposent avec les touristes. Les habitants de pôles touristiques cubains mettent ainsi en avant "l'interaction sociale avec le monde extérieur " (Colantonio \& Potter, 2006: 163) que sous-tend le tourisme, et voient bien dans la visite d'étrangers la possibilité d'établir un véritable échange avec d'autres cultures. On observe dès lors que ce dernier peut être à la base d'échanges entre les cultures cubaine et mondiale, et se convertir alors en clé du dialogue de lîle avec le monde extérieur.

En outre, on considère que ces échanges entre touristes et Cubains peuvent aider ces derniers à revendiquer plus de liberté, puisque l'arrivée d'étrangers induit un contact entre cultures, et une ouverture sur les différents modes de gouvernement. Partant, le tourisme est non seulement une porte d'entrée pour différentes idées, mais aussi une fenêtre ouverte sur une coopération entre Cubains et le monde, permettant aux habitants de l'île d'asseoir leurs revendications démocratiques, sans pour autant adopter un mode de vie qui n'est pas le leur. Ainsi, si comme nous l'avons déjà dit, le tourisme peut constituer un danger pour le gouvernement de l'île, il peut également être un pilier à la construction d'une nouvelle société pour la population.

\section{Conclusion}

39 Notre analyse nous pousse à dire que le tourisme semble à la fois être un mal et un remède pour l'île. Dans les pays d'origine des visiteurs, la Révolution demeure une nébuleuse, tantôt romantique tentative de quelques barbudos en mal d'aventure, tantôt piège d'où personne ne s'échappe, et les guides touristiques, tout comme les médias, contribuent à diffuser de nombreux clichés concernant Cuba dont il est difficile de se départir lorsque l'on arrive dans l'île. Malgré tout, et peut-être précisément du fait qu'elle reste un mystère, Cuba attire. Les autorités l'ont bien compris, qui promeuvent une offre touristique adaptée à la demande, déclinant tous les atouts de l'île en autant d'offres de séjour. Mais si l'ouverture de Cuba au tourisme s'est faite dans la douleur, et dans le but de sauver une économie ravagée par la perte de l'allié soviétique, l'adaptation de la population à un nouveau contact avec l'extérieur n'a pas non plus été sans mal.

Ainsi, les tourismes proposés par le Crocodile vert constituent des chances pour l'économie du pays, tout en se présentant comme des dangers pour sa culture, l'identité de son peuple, et même son système politique. Face aux visiteurs, les Cubains se voient dans l'obligation de dresser un bilan des acquis de la Révolution, qui, s'ils sont indéniables, ne les encouragent plus nécessairement à soutenir un système qui a montré ses failles, n'a pas empêché la réapparition de différences socio-économiques, et conduit même à traiter de façon inéquitable touristes et habitants. En outre, la loi de l'offre et de la demande dirige parfois la production culturelle et artisanale de l'île, faisant douter des bénéfices du tourisme concernant l'identité des Cubains.

41 Le dialogue et le contact entre visiteurs et Cubains n'ont pas nécessairement été très encouragés, qu'on ait craint la propagation d'idées contre-révolutionnaires chez ses derniers, ou que l'on ait eu à cœur de circonscrire le jineterismo. Finalement, le voyageur, qu'il le veuille ou non, laisse à Cuba une trace, même s'il reste à savoir si cette empreinte contribue à une évolution ou à un changement définitif, une mutation de la société 
cubaine. S'il s'en donne la peine, il peut en outre revenir de la plus grande des Antilles avec bien d'autres choses que les clichés attendus.

\section{BIBLIOGRAPHIE}

Auzias Dominique \& Labourdette Jean-Paul (2010), Le Petit Futé - Cuba, Paris : Nouvelles Éditions de l'Université.

Auzias Dominique \& LABourdetTe Jean-Paul (2016), Le Petit Futé - Cuba, Nouvelles Éditions de l'Université, édition numérique.

BASTIAN Jean-Pierre (2007), « Les usages réciproques du tourisme et de la religion dans une logique de marché », J. - P. Bastian (dir.), Pluralisation religieuse et logique de marché, Berne : Peter Lang, 157-174.

COLANTONIO Andrea \& POTTER Robert (2006), Urban tourism and development in the socialist state: Havana during the "Special period", Ashgate : Aldershot.

GAY-SYLVESTRE Dominique \& EstRADE Paul (2006), Être femme à Cuba : des premières militantes féministes aux militantes révolutionnaires, Paris : L'Harmattan.

GÉOGUIDE CUBA (2014), Gallimard Loisirs, édition numérique.

Goldstone Patricia, MoRAles Vidal Andrea \& BASTida Xohana (2003), Turismo: más allá del ocio y del negocio, Barcelone : Debate.

LAMIC Jean-Pierre (2008), Tourisme durable : utopie ou réalité ? Comment identifier les voyageurs et voyagistes éco-responsables?, Paris : L'Harmattan.

LEROUX MONET Rémy (2008), Cuba, carnets de route, Paris : Marcus.

MicheL Franck (1998), « Le tourisme international : une bouée de sauvetage pour Cuba? », F. Michel (dir.), Tourismes, touristes, sociétés, Paris : L'Harmattan, 251-288.

Michel Frank (2006), Voyage au bout du sexe : trafics et tourisme sexuels en Asie et ailleurs, SainteFoix : Les Presses de l'Université de Laval.

LEMIEUX Hector (2013), Comprendre Cuba, Montréal : Ulysse.

RABOTEUR Joël (2009a), « La nouvelle carte touristique de la Caraïbe : quelle place pour la Guadeloupe ?", P. Roselé Chim et al.(dir.), Le développement du tourisme de santé, de remise en forme et de bien-être, Paris : Publibook.

RABOTEUR Joël (2009b), «Impact économique de la culture dans le développement touristique », P. Roselé Chim et al.(dir.), Le développement du tourisme de santé, de remise en forme et de bien-être, Paris : Publibook.

SIMONI Valerio (2011), «L'interculturalité comme justification : sexe "couleur locale" dans la Cuba touristique », A. Lavanchy, A. Gajardo \& F. Dervin (dir.), Anthropologies de l'interculturalité, Paris : L'Harmattan, 197-226. 


\section{ANNEXES}

Janice Argaillot est docteur en études latino-américaines (Université de Cergy-Pontoise, 2011), maître de conférences en civilisation latino-américaine et langue espagnole de spécialité (Université Grenoble-Alpes, ILCEA4) depuis septembre 2013. Codirectrice du centre délocalisé pour l'université Stendhal de janvier 2015 à janvier 2016, Janice Argaillot est responsable de la Licence professionnelle Métiers du Commerce International (Université Grenoble-Alpes) depuis le $1^{\mathrm{er}}$ septembre 2016. Ses activités de recherche et ses publications portent sur Cuba, les Caraïbes et l'Amérique latine contemporaine.

\section{NOTES}

1. <http://www.one.cu/publicaciones/06turismoycomercio/llegadadevisitantes/mensual/2.pdf>

2. <http://www.one.cu/aec2011/esp/15_tabla_cuadro.htm>

\section{RÉSUMÉS}

Cet article propose une analyse du tourisme et de ses impacts à Cuba. En effet, la plus grande île de la Caraïbe offre aux visiteurs de nombreuses façons de la découvrir, mais il n'est pas certain que tous les voyageurs puissent sortir des sentiers battus pour saisir cette opportunité. En outre, la multiplicité de l'offre touristique a induit de nombreuses conséquences pour le peuple cubain, économiques bien sûr, mais également dans les champs politique, social et sociétal. Ce travail s'attache donc à comprendre les répercussions de l'ouverture de l'île au tourisme sur sa population, en cherchant à savoir si le voyageur ne fait que passer sur le territoire cubain, ou s'il s'y ancre durablement.

This paper aims at analyzing tourism and its different impact in Cuba. Indeed, the largest Caribbean island offers visitors many ways of discovering it, but it is not sure that all travelers could escape from the beaten path to seize this opportunity. Moreover, the diversity of the touristic offer has had a lot of consequences on the Cuban people: economic, political, as well as social and societal consequences. Ultimately this leads us to wonder whether travelers are only passing on Cuban territory, or if they settle on it more permanently.

\section{INDEX}

Mots-clés : Cuba, crise, tourisme, culture

Keywords : Cuba, crisis, tourism, culture 
AUTEUR

JANICE ARGAILLOT

Maître de conférences, Université Grenoble-Alpes (ILCEA4) 